PROCEEDINGS OF THE

AMERICAN MATHEMATICAL SOCIETY

Volume 128, Number 4, Pages 1039-1045

S 0002-9939(99)05040-6

Article electronically published on July 28, 1999

\title{
ON AUTOMATIC CONTINUITY OF HOMOMORPHISMS
}

\author{
A. BEDDAA, S. J. BHATT, AND M. OUDADESS
}

(Communicated by Dale Alspach)

\begin{abstract}
Introducing a weaker notion of regularity in a topological algebra, we examine and improve an automatic continuity theorem given by the second author. Examples and applications are given.
\end{abstract}

All topological algebras considered are commutative and Hausdorff, having a unit element. A topological algebra $A$ is a $Q$-algebra if the set $G(A)$ of invertible elements is open. Let $B$ be a subalgebra of an algebra $A$. Then $B$ is inverse closed in $A$ if $G(B)=B \cap G(A)$. $A$ is strongly semisimple if for every $x \in A, x \neq 0$, there exists a nonzero continuous multiplicative linear functional $\chi$ such that $\chi(x) \neq 0$. $A$ is advertibly complete if a Cauchy net $x_{\alpha}$ in $A$ converges in $A$ whenever for some $y$ in $A, x_{\alpha}+y-x_{\alpha} y$ converges to 0 . A $Q$-algebra is advertibly complete [Ma, p. 45]. A uniform seminorm on an algebra $A$ is a seminorm $p$ such that $p\left(x^{2}\right)=p(x)^{2}$ for all $x$ in $A$. Such a $p$ is submultiplicative [BK]. A uniform topological algebra $A$ is a topological algebra whose topology is defined by a family of uniform seminorms. Such an $A$ is semisimple. The abbreviation lmca will stand for locally $m$-convex algebra.

In $[\mathrm{B}$, the following is given.

Theorem ([B, Theorem 2.2]). Let $A$ be a spectrally bounded, regular, complete, uniform topological algebra. If $B$ is an lmca and $\phi: A \rightarrow B$ is a one-to-one homomorphism such that $(\operatorname{Im} \phi)^{-}$(the closure of $\left.\operatorname{Im} \phi\right)$ is a semisimple Q-algebra, then $\phi^{-1} / \operatorname{Im} \phi$ is continuous.

In the proof, the author considers the map $\phi^{*}: \sigma(C) \rightarrow \sigma(A)$, with $\phi^{*}(f)=f \circ \phi$, $\sigma(C)$ and $\sigma(A)$ denoting respectively the spaces of nonzero continuous multiplicative functionals on $C=(\operatorname{Im} \phi)^{-}$and $A$. In Math. Reviews, the reviewer R. J. Loy [L] asserted that the continuity of $\phi$ has been implicitly used in [B]. Indeed, $\phi^{*}$ is not always well defined when $\phi$ is not continuous as the following example shows.

Example 1. Let $\Omega$ denote the first uncountable ordinal and $[0, \Omega)$ the set of all ordinals smaller than $\Omega$. Consider the algebra $C[0, \Omega)$ of complex continuous functions on $[0, \Omega)$ with compact open topology $\tau$. Every $f \in C[0, \Omega)$ is bounded. It is a regular uniform lmca. The identity map $\phi:(C[0, \Omega), \tau) \rightarrow\left(C[0, \Omega),\|\|_{\infty}\right), \phi(f)=f$, satisfies the hypotheses of the above statement. It is well known that $(C[0, \Omega), \tau)$ has discontinuous multiplicative linear functionals (see, for example, [Mi], [Z]); let $\chi$

Received by the editors September 10, 1997 and, in revised form, May 18, 1998.

1991 Mathematics Subject Classification. Primary 46H40; Secondary 46H05.

Key words and phrases. Uniform topological algebra, locally $m$-convex algebra, weakly regular algebra, advertibly complete algebra, $Q$-algebra, automatic continuity of homomorphism. 
be such a functional. It is in $\sigma\left(C[0, \Omega),\|\|_{\infty}\right)$, but $\phi^{*}(\chi)=\chi$ is not in $\sigma([0, \Omega), \tau)$. One may give another example in a more general situation. Let $X$ be a noncompact, locally compact pseudocompact space. Take $A=C(X)$ the algebra of all continuous functions on $X$ with compact open topology, $B$ the algebra $C(X)$ endowed with the sup norm \|\|$_{\infty}$ and $\phi: A \rightarrow B$ be $\phi(f)=f$. Since $X$ is pseudocompact, non-compact, it is not realcompact $[\underline{\mathrm{S}}$, p. 44]. Let $y$ be an element of the real compactification of $X$ with $y$ not in $X$. The evaluation $\delta_{y}$ at $y$ is in $\sigma(B)$ but not in $\sigma(A)$. Hence $\phi^{*}$ is not defined on $\delta_{y}$.

On the other hand, $\phi^{*}$ can be well defined even when $\phi$ is not continuous; in this case, the proof given in [B] works. Here is an example of such a situation.

Example 2. Let $X$ be a compact Hausdorff space. Consider the algebra $C(X)$ of continuous complex functions on $X$. Take $A=\left(C(X), \tau_{d}\right), \tau_{d}$ being the topology of uniform convergence on all countable compact subsets of $X, B$ the algebra $\left(C(X),\|\|_{\infty}\right)$ and $\phi$ the identity map from $A$ to $B$. It is of course discontinuous, only if $X$ is uncountable. But in this case, $\sigma(A)$ and $\sigma(B)$ are both homeomorphic to $X$. So $\phi^{*}$ is well defined.

The following theorem repairs the above result. On one hand, it provides a positive result in a context more general than above; on the other hand, it shows that if one assumes the continuity of $\phi$ in the above result, then the algebra $A$ is necessarily a Banach algebra.

Theorem 1. Let $A$ be weakly regular, advertibly complete, uniform topological algebra, let $B$ be an lmca, and let $\phi: A \rightarrow B$ be a one-to-one homomorphism such that $(\operatorname{Im} \phi)^{-}$is a semisimple $Q$-algebra.

(1) If $A$ is functionally continuous, then $\phi^{-1} / \operatorname{Im} \phi$ is continuous.

(2) If $\phi$ is continuous, then the topology of $A$ is normable.

Following [Mi, p. 51], $A$ is functionally continuous $(F C)$ if every multiplicative functional on $A$ is continuous. Note that $\left(C(X), \tau_{d}\right)$ in Example 2 is $\mathrm{FC}$, but not $Q$. A major unsolved problem in topological algebras is the Michael problem: Is every multiplicative linear functional on a Frechet lmc algebra continuous? This has led to several sufficient conditions for $A$ to be FC. This makes FC a reasonable assumption.

Let $A$ be a commutative topological algebra. $A$ is weakly regular if given a closed set $F \subset \sigma(A), F \neq \sigma(A)$, there exists $x \neq 0$ in $A$ such that $f(x)=0$ for all $f \in F$. In the context of Banach algebras, weak regularity arises naturally in the study of uniqueness of the uniform norm [BD]; and it is weaker than regularity. This is exhibited in an example due to Barnes [Me, Example 1]. Let $D=\{z \in C:|z|<1\}$, $X=\bar{D} \times[0,1]$. Let $A=\{f \in C(X): f$ is holomorphic on $D \times\{0\}\}$, a uniform Banach algebra. Then $A$ is weakly regular, but not regular. Since regularity in a uniform algebra is a stringent property, the validity of Theorem 1 under weak regularity is interesting.

Proof of Theorem 1] (1) Let $C=(\operatorname{Im} \phi)^{-}$. Since $A$ is FC, $\phi^{*}: \sigma(C) \rightarrow \sigma(A)$, $\phi^{*}(f)=f \circ \phi$ is well defined. It is also continuous with respective Gelfand topologies. Since the algebra $C$ is a locally convex $Q$-algebra, $\sigma(C)$ is compact [Ma, p. 187]. Then $\phi^{*}(\sigma(C))$ is a compact subset of $\sigma(A)$. We have $\phi^{*}(\sigma(C))=\sigma(A)$. Indeed, if $\phi^{*}(\sigma(C)) \neq \sigma(A)$, there exists $x \neq 0$ in $A$ such that $\chi(\phi(x))=0$ for all $\chi$ in $\sigma(C)$. 
Since $C$ is commutative semisimple and lmc, $\phi(x)=0$; and then $x=0$ for $\phi$ is oneto-one. Thus $\phi^{*}(\sigma(C))=\sigma(A)$. Now let $P=\left(p_{\alpha}\right)$ be a family of uniform seminorms defining the topology $\tau$ of $A$. Since $A$ is advertibly complete, the spectrum $\operatorname{Sp}_{A}(x)=$ $\{\chi(x): \chi \in \sigma(A)\}$ and the spectral radius $\rho_{A}(x)=\sup _{\alpha}\left\{\lim _{n \rightarrow \infty}\left(p_{\alpha}\left(x^{n}\right)\right)^{1 / n}\right\}$ for all $x$ in $A$ [Ma, p. 104, p. 99]. Since $\sigma(A)$ is compact, $\operatorname{Sp}_{A}(x)$ is bounded. Since $p_{\alpha}\left(x^{2}\right)=p_{\alpha}(x)^{2}$ for all $x$ and $\alpha, \rho_{A}(x)=\sup _{\alpha} p_{\alpha}(x)$ for all $x \in A$. Also, $\sigma(A)=\phi^{*}(\sigma(C))$ gives $\operatorname{Sp}_{A}(x)=\{f(\phi(x)): f \in \sigma(C)\} \subset \operatorname{Sp}_{C}(\phi(x))$. Further, as $C$ is a $Q$-algebra, $s(C)=\left\{x \in C: \rho_{C}(x) \leq 1\right\}$ is a neighbourhood of 0 by [Mi, Prop. 13.5, p. 58]; and there exists a convex balanced open set $W$ such that $0 \in W \subseteq s(C)$. The Minkowski functional $q$ of $W$ in $C$ is a continuous seminorm satisfying $\rho_{C}(y) \leq q(y)$ for all $y \in C$. Hence for each $\alpha$, for each $x \in A, p_{\alpha}(x) \leq$ $\rho_{A}(x) \leq \rho_{C}(\phi(x)) \leq q(\phi(x))$. This proves that $\phi^{-1} / \operatorname{Im} \phi$ is continuous.

(2) Suppose $\phi$ is continuous. Then $\phi^{*}: \sigma(C) \rightarrow \sigma(A)$ is well defined even if $A$ is not FC. Then $\phi^{-1} / \operatorname{Im} \phi$ is continuous as above making $\phi$ a topological isomorphism. Thus $\phi(A)$ is advertibly complete; and hence inverse closed in its completion. Whence it is inverse closed in the $Q$-algebra $C$, for the completion of $\phi(A)$ is contained in $C$. Therefore $\phi(A)$, and so $A$, is a $Q$-algebra. Hence the topology on $A$ given by the algebra norm $\rho_{A}$ is finer than $\tau$. Now since $A$ is a $Q$-algebra, $s(A)=\left\{x \in A: \rho_{A}(x) \leq 1\right\}$ is a neighbourhood of 0 on $(A, \tau)$. Thus $\rho$ determines $\tau$.

Remark. Once $\phi^{*}$ is defined, the full strength of weak regularity has not been used. In fact, one has only to find a nonzero element vanishing on a given compact set.

We now give a result in the absence of FC. We consider the space $\sigma^{*}(A)$ consisting of all nonzero multiplicative functionals on $A$ endowed with the weak topology $\sigma\left(A^{*}, A\right)$. We then introduce the following notion of weak $\sigma^{*}$-compact-regular weakened in the sense of the previous remark.

Definition. A commutative topological algebra $A$ is called weakly $\sigma^{*}$-compactregular if for a compact subset $K$ of $\sigma^{*}(A), K \neq \sigma^{*}(A)$, there exists a nonzero $x \in A$ such that $\chi(x)=0$ for all $\chi \in K$.

Theorem 2. Let $A$ be a weakly $\sigma^{*}$-compact-regular advertibly complete uniform algebra, $B$ a locally convex algebra and $\phi: A \rightarrow B$ a one-to-one homomorphism such that $C=(\operatorname{Im} \phi)^{-}$is a strongly semisimple $Q$-algebra. Then $\phi^{-1} / \operatorname{Im} \phi$ is continuous. If $\phi$ is continuous, then the topology of $A$ is normable.

For the proof, consider the map $\phi^{* *}: \sigma(C) \rightarrow \sigma^{*}(A), \phi^{* *}(\chi)=\chi \circ \phi$. If $\chi \circ \phi$ is identically zero, then by the continuity of $\chi$, one obtains that $\chi$ is also identically zero. This contradicts $\chi \in \sigma(C)$. Thus $\phi^{* *}$ is defined; and then it is continuous. Now one obtains $\phi^{* *}(\sigma(C))=\sigma^{*}(A)$; and the proof can be completed as in Theorem 1

We conjecture that the semisimplicity of $(\operatorname{Im} \phi)^{-}$in Theorem 1 (and strong semisimplicity in Theorem 21) can be omitted. The following supports this.

Theorem 3. Let $A$ be a uniform lmca, $B$ a locally convex algebra, and $\phi: A \rightarrow B$ a one-to-one homomorphism such that $(\operatorname{Im} \phi)^{-}$is a $Q$-algebra. Assume that at least one of the following holds.

(a) $A$ is advertibly complete and $\operatorname{Im} \phi$ is FC with continuous product.

(b) A is FC, Ptak (as a l.c. space), regular, having locally equicontinuous spectrum $\sigma(A)$ (in particular, $A$ is FC, Frechet, regular, having locally compact spectrum 
$\sigma(A))$, and $B$ is lmca. Then $\phi^{-1} / \operatorname{Im} \phi$ is continuous. If $\phi$ is continuous, then the topology of $A$ is normable.

Proof. (1) Assume (a). Then $\sigma^{*}\left((\operatorname{Im} \phi)^{-}\right)=\sigma\left((\operatorname{Im} \phi)^{-}\right)$(since a $Q$-algebra is $\mathrm{FC})=\sigma(\operatorname{Im} \phi)$ (by the joint continuity of multiplication in $\operatorname{Im} \phi)=\sigma^{*}(\operatorname{Im} \phi)$ and $\phi^{*}\left(\sigma^{*}(\operatorname{Im} \phi)\right)=\sigma^{*}(A)$ as $\phi$ is one-to-one. Then, for all $x \in A, \operatorname{Sp}_{A}(x)=\{\chi(x): \chi \in$ $\sigma(A)\}=\left\{\chi(x): \chi \in \sigma^{*}(A)\right\}=\left\{f(\phi(x)): f \in \sigma^{*}(\operatorname{Im} \phi)\right\}=\left\{f(\phi(x)): f \in \sigma^{*}(C)\right\}$. Hence for some continuous seminorm $q, \rho_{A}(x)=\rho_{C}(x) \leq q(\phi(x))(x \in A)$.

(2) Assume (b). By [Ma, Coro. 1.3, p. 184], local equicontinuity of $\sigma(A)$ implies continuity of the Gelfand map $x \rightarrow \hat{x}$ and local compactness of $\sigma(A)$. We show that $\sigma(A)=\phi^{*}(\sigma(C))$. Note that $\phi^{*}(\sigma(C)) \subset \sigma(A)$. Suppose $\chi \in \sigma(A) \backslash \phi^{*}(\sigma(C))$. By the local compactness, there exists a compact set $K \subseteq \sigma(A)$ and disjoint open sets $U, V$ in $\sigma(A)$ such that $\chi \in K \subset U, \phi^{*}(\sigma(C)) \subset \bar{V}$. As $A$ is Ptak, regular and having continuous Gelfand map, [Ma, Coro. 4.4, p. 344] implies that there exist $x, y \in A$ such that $g(x)=1\left(g \in \phi^{*}(\sigma(C))\right), g(x)=0(g \in \sigma(A) \backslash V)$; $g(y)=1(g \in K), g(y)=0(g \in \sigma(A) \backslash U)$. Then $g(x) g(y)=0$ for all $g \in \sigma(A)$. By the semisimplicity of $A, x y=0=\phi(x) \phi(y)$. On the other hand, for all $f \in \sigma(C)$, $f(\phi(x))=1$. Thus $0 \notin\{f(\phi(x)): f \in \sigma(C)\}=\operatorname{Sp}_{C}(\phi(x)), C$ being lmc and a $Q$-algebra. Thus $\phi(x)$ is invertible in $C$. Hence $\phi(y)=\phi(x)^{-1} \phi(x) \phi(y)=0$, so that $y=0$, a contradiction. It follows that $\phi^{*}(\sigma(C))=\sigma(A)$. Now the proof can be completed as in Theorem 11. Note that if $A$ is Frechet, then every compact subset of $\sigma(A)$ is equicontinuous [Mi, Prop. 4.2, p. 17]. Hence by [Ma, Th. 1.1, p. 182], the Gelfand map is continuous. Further if $\sigma(A)$ is locally compact, then it is locally equicontinuous [Ma, Cor. 1.3, p. 184].

Remarks. (1) If $B$ is lmca and $C=(\operatorname{Im} \phi)^{-}$is a semisimple $Q$-algebra, then $C$ is strongly semisimple.

(2) Actually in the above theorems, $\phi^{-1}: \phi(A) \rightarrow(A,\|\|)$ is continuous, where \|\| is the uniform norm given by $\|x\|=\sup \{p(x): p$ is a continuous uniform seminorm $\}$. The existence of this norm implies that $A$ is spectrally bounded.

(3) Theorem 2 also applies to Example 1. Indeed, the algebra $(C[0, \Omega), \tau)$ is a complete uniform algebra. It is weakly $\sigma^{*}$-compact-regular, for $\sigma^{*}(C[0, \Omega))$ is homeomorphic to the Stone-Cech compactification $\beta[0, \Omega)$ of $[0, \Omega)$ and $C[0, \Omega)$ is isomorphic to the algebra $C(\beta[0, \Omega))$ GJ].

(4) The hypothesis $(\operatorname{Im} \phi)^{-}$is a $Q$-algebra cannot be omitted. Let $A=C_{b}(\mathbb{R})$ be the algebra of all continuous bounded functions on the real line. Endowed with the sup norm, it is a uniform Banach algebra. By the same arguments as in (3), one shows that $A$ is weakly $\sigma^{*}$-compact-regular. Consider $B=C(\mathbb{R})$ to be the algebra of all continuous functions with the compact open topology. Consider $\phi: A \rightarrow B$, $\phi(f)=f$. Then $(\operatorname{Im} \phi)^{-}=C(\mathbb{R})$. It is well known that it is not a $Q$-algebra. Clearly $\phi^{-1}$ is discontinuous.

(5) The referee has asked: (In above theorems) does the automatic continuity of $\phi^{-1}$ on $\operatorname{Im} \phi$ necessitate $(\operatorname{Im} \phi)^{-}$a $Q$-algebra? The following answers this.

Proposition 4. Let $A$ be a complete $Q$-lmca, $B$ an lmca, and $\phi: A \rightarrow B$ a one-toone homomorphism such that $\phi^{-1} / \operatorname{Im} \phi$ is continuous. Then $(\operatorname{Im} \phi)^{-}$is a $Q$-algebra.

Proof. We may assume that $B$ is complete, hence $C=(\operatorname{Im} \phi)^{-}$is complete. Then $\phi^{-1} / \operatorname{Im} \phi$ extends as a continuous homomorphism $\psi: C \rightarrow A$. By assumption, there exists a continuous seminorm $p$ on $A$ such that for all $x$ in $A, r_{C}(\phi(x)) \leq$ $r_{\operatorname{Im} \phi}(\phi(x)) \leq r_{A}(x) \leq p(x) \leq p(\psi(\phi(x)))$. Since $\psi$ is continuous, there exists a 
continuous seminorm $q$ on $C$ such that $p(\psi(y)) \leq q(y)(y \in C)$. Hence in the above, $r_{C}(\phi(x)) \leq p(\psi(\phi(x))) \leq q(\phi(x))$ for all $x$ in $A$. Now let $y \in C, y=\lim \phi\left(x_{\alpha}\right)$ for some net $\left(x_{\alpha}\right)$ in $A$. For any continuous multiplicative functional $f$ on $C$, $|f(y)| \leq\left|f\left(y-\phi\left(x_{\alpha}\right)\right)\right|+\left|f\left(\phi\left(x_{\alpha}\right)\right)\right| \leq\left|f\left(y-\phi\left(x_{\alpha}\right)\right)\right|+q\left(\phi\left(x_{\alpha}\right)\right) \rightarrow q(y)$. Hence $r_{C}(y)=\sup |f(y)| \leq q(y)(y \in C)$ showing that $C$ is a $Q$-algebra.

If $A$ is not a $Q$-algebra, then this does not hold. For the open unit disc $U$ in the complex plane, let $A=H(U)$ be the uniform Frechet algebra consisting of holomorphic functions on $U$ with the compact-open topology, $B=C(U)$ with the compact-open topology, and $\phi: A \rightarrow B$ be $\phi(f)=f$. Clearly $\phi$ is a homeomorphism and $(\operatorname{Im} \phi)^{-}=B$ fails to be a $Q$-algebra.

\section{Applications}

(1) Proposition. Let $A$ be an advertibly complete lmca. Let \|\| be any continuous norm on $A$. Then $A$ cannot be simultaneously weakly regular and uniform unless the topology of $A$ is normable.

Proof. Let $\tau$ denote the lmc topology on $A$. Let $P=\left(p_{\alpha}\right)$ be a family of submultiplicative seminorms on $A$ defining $\tau$. Then $P_{0}=P \cup\{\|\|\}$ also determines $\tau$. Suppose $(A, \tau)$ is uniform. Then $\tau$ is defined by a family $S=\left(q_{i}\right)$ of uniform seminorms. By closing $S$ with maxima of finite subfamilies and applying the continuity of \|\| , there exists a $q$ in $S$ which is a norm. Let $A_{q}$ be the uniform Banach algebra obtained by completing $(A, q)$. Let $\phi: A \rightarrow A_{q}$ be $\phi(x)=x$. Now if $A$ is weakly regular, then Theorem 1 applied to $\phi$ implies that $\tau$ is normable.

It follows that an advertibly complete non-normed weakly regular uniform algebra cannot support a continuous norm. Let $X$ be a compact Hausdorff space. By a well known result of Kaplansky, if || is any norm on $C(X)$ making it a normed algebra, then the supnorm \|\|$\leq||$. The following has a bearing with this. A norm on an algebra $A$ is semisimple if the completion of $(A,||)$ is semisimple $\mathrm{BD}$.

(2) Corollary. Let \|\| be a uniform norm on an algebra $A$ such that $(A,\|\|)$ is a weakly regular $Q$-normed algebra. Let || be any submultiplicative norm on $A$.

(i) If || is semisimple, then \|\|$\leq||$. Further if || is continuous, then || is equivalent to \|\| .

(ii) Let $(A,\|\|)$ be complete and regular. Then \|\|$\leq \mid$ for any submultiplicative norm $\mid$.

Indeed let $\phi:(A,\|\|) \rightarrow(\widetilde{A},||)(\widetilde{A}=$ completion of $(A,||)), \phi(x)=x$. Theorem पimplies that there exists $k>0$ such that \|\|$\leq k||$. Since $(A,\|\|)$ is $Q, \rho_{A}(x)=$ $\inf \left\|x^{n}\right\|^{1 / n}=\lim \left\|x^{n}\right\|^{1 / n}=\|x\| \leq \lim \left|x^{n}\right|^{1 / n} \leq|x|$ for all $x \in A$. (ii) follows by Theorem $3(\mathrm{~b})$.

(3) Let $A=\mathbb{C} \times C_{c}^{\infty}(\mathbb{R})$ (resp. $B=\mathbb{C} \times C_{c}(\mathbb{R})$ ) be the algebra of all complex $C^{\infty}$-functions (resp. continuous functions) on $\mathbb{R}$ which are constant outside some compact set (depending on the function). We endow $A$ (resp. $B$ ) with the inductive limit topology $\tau_{D}$ (resp. $\left.\tau_{K}\right)$. The algebra $\left(A, \tau_{D}\right)$ is a complete regular lmca, $\left(B, \tau_{K}\right)$ is a lmc $Q$-algebra [Ma, p. 128] and $A$ is dense in $B$ [K, p. 148]. Consider $\phi: A \rightarrow B, \phi(f)=f$. Since the topology $\tau_{D}$ is finer than $\tau_{K}$ on $A, \phi$ is continuous. It is classical that $A$ is not normable. Hence by Theorem 1 , $\left(A, \tau_{D}\right)$ cannot be uniform.

Let $A$ be the algebra $\mathbb{C} \times C_{c}^{\infty}(\mathbb{R})$ endowed with the compact open topology $\tau$. It is a weakly $\sigma^{*}$-compact-regular uniform lmca. Since it is inverse closed in $C(\mathbb{R})$, it 
is advertibly complete. Take $\left(B, \tau_{K}\right)$ as above. Let $\phi:(A, \tau) \rightarrow\left(B, \tau_{K}\right), \phi(f)=f$. By Theorem 1, $\phi^{-1}$ is continuous.

(4) Let $U \subset \mathbb{C}^{d}$ be open. Let $H(U)$ be the uniform Frechet algebra of all holomorphic functions on $U$ with the compact open topology. Let $H^{\infty}(U)=\{f \in$ $H(U): f$ is bounded $\}$, a uniform Banach algebra. Let $X \subset \mathbb{C}^{d}$ be compact. Let $H(K)$ be the algebra of holomorphic germs on $X$. Choose a decreasing sequence $\left(U_{n}\right)$ of open neighbourhoods of $X$ such that $\bar{U}_{n+1} \subset U_{n}$ and $\bar{U}_{n+1}$ is compact. In view of the continuous embeddings

$$
\cdots \rightarrow H^{\infty}\left(U_{n}\right) \rightarrow H\left(U_{n}\right) \rightarrow H^{\infty}\left(U_{n+1}\right) \rightarrow H\left(U_{n+1}\right) \rightarrow \cdots,
$$

$H(X)$ can be realized as inductive limits $H(X)=\lim H\left(U_{n}\right)=\lim H^{\infty}\left(U_{n}\right)$, its topology $\tau$ being the finest locally convex topology making all $\phi_{n}: \overrightarrow{H\left(U_{n}\right)} \rightarrow H(X)$, $\phi_{n}(f)=f / X$ and similarly making all $\psi_{n}: H^{\infty}\left(U_{n}\right) \rightarrow H(X), \psi_{n}(f)=f / X$ continuous.

None of $H^{\infty}(U)$ and $H(U)$ is weakly regular. Note that $(H(X), \tau)$ is a complete semisimple $Q$-algebra [Ma, p. 134]. If $H\left(U_{n}\right)$ is weakly regular, then by Theorem 11 it becomes a Banach algebra and $\phi_{n}$ becomes a homeomorphism. If $H^{\infty}\left(U_{n}\right)$ is weakly regular, then $\psi_{n}$ becomes a homeomorphism. Either of these forces $H(X)$ to be a uniform Banach algebra. Being a complete, non-normed $Q$-algebra, $H(X)$ is not a uniform algebra $[\mathrm{BD}]$.

(5) Let $D=\{z \in \mathbb{C}:|z|<1\}, Y=D \times[0,1], Z=\bar{D} \times[0,1)$. Let $A=\{f \in$ $C(Y): f$ is holomorphic on $D \times\{0\}\}, B=\{f \in C(Z): f$ is holomorphic on $D \times$ $\{0\}\}$. Let $0<r<1$. Let $\|f\|_{r}=\sup \left\{|f(x)|: x \in \bar{D}_{r} \times[0,1]\right\}(f \in A) ;|f|_{r}=$ $\sup \{|f(x)|: x \in \bar{D} \times[0, r]\}(f \in B)$. Each of $A$ and $B$ with the topology defined respectively by $\left\{\|\|_{r}: 0<r<1\right\}$ and $\left\{||_{r}: 0<r<1\right\}$ is a uniform Frechet algebra. Any $f \in C(Y)$ (resp. $f \in C(Z)$ ) vanishing on $D \times\{0\}$ is in $A$ (resp. in $B$ ). This implies that both $A$ and $B$ are weakly regular, not regular. Thus each of $A$ and $B$ fails to support a continuous norm.

\section{ACKNOWLEDGEMENT}

A query by the referee resulted in Proposition 4. The referee has also made several suggestions regarding the exposition. We sincerely thank the referee for these.

\section{REFERENCES}

[BK] S. J. Bhatt and D. J. Karia, Uniqueness of the uniform norm with an application to topological algebras, Proc. Amer. Math. Soc. 116 (2) (1992), 499-503. MR 92m:46068

[B] S. J. Bhatt, Automatic continuity of homomorphisms in topological algebras, Proc. Amer. Math. Soc. 119 (1) (1993), 135-139. MR 93k:46042

[BD] S. J. Bhatt and H. V. Dedania, Banach algebras with unique uniform norm, Proc. Amer. Math. Soc. 124 (2) (1996), 579-584. MR 96d:46067

[GJ] L. Gillman and M. Jerison, Rings of continuous functions, Springer-Verlag, New York, Heidelberg, Berlin, 1976. MR 53:11352

[K] Vo-Khac Khoan, Distributions Analyse de Fourier Operateurs aux dérivees partielles, vol. T. 1, Vuibert, Paris, 1972.

[L] R. J. Loy, Math. Reviews, 93k:46042, p. 6116, Amer. Math. Soc., 1993.

[Ma] A. Mallios, Topological Algebras, Selected Topics, North-Holland, 1986. MR 87m:46099

[Me] M. J. Meyer, Spectral extension property and extension of multiplicative linear functionals, Proc. Amer. Math. Soc. 112 (1991), 855-861. MR 91j:46059

[Mi] E. Michael, Locally multiplicatively-convex algebras, Mem. Amer. Math. Soc., vol. 11, Providence, RI, 1952. MR 14:482a 
[S] J. Schmets, Espaces de fonctions continues, Lecture Notes in Mathematics 519, SpringerVerlag, 1976. MR 54:11041

[Z] W. Zelazko, Banach algebras, Elsevier Publishing Co. Amsterdam, London, New York, 1973. MR 56:6389

(A. Beddaa and M. Oudadess) Ecole Normale Superieure, B.P. 5118 Takaddoum, Rabat, MOROC

(S. J. Bhatt) Department of Mathematics, Sardar Patel University, Vallabh VidyanaGAR 388120, GuJArat, India 